\title{
Shielded Electronic Current Transformer
}

\author{
Daniel Slomovitz, Senior Member, IEEE, and Heriguatí de Souza
}

\begin{abstract}
A current transformer with nominal ratio 10 A to $10 \mathrm{~mA}$, intended for low-frequency applications, was developed. It includes an electronic device to reduce the magnetizing current, and a continuous shield in the secondary winding (coaxial cable) in order to eliminate the effect of stray capacitances. No guard-source is connected to the shield. It is proposed in this paper to leave the cable-shield-potential floating. This leads to high-accuracy results (ratio errors and phase-displacements in the order of few parts in $10^{6}$ from $50 \mathrm{~Hz}$ to $1 \mathrm{kHz}$ ).
\end{abstract}

Index Terms-Compensation, current, error, guard, measuring transformer, phase displacement, power frequency, shield.

\section{INTRODUCTION}

$\mathbf{M}$ ANY electronic instruments, e.g., power-energy standards, require a current to voltage transducer with low ratio and phase-displacements errors. Input current range is typically from 1-10 A, for high-precision meters, and the output voltage of the current channel must be around $1 \mathrm{~V}$. If a shunt resistor was used, its value would be $0.1 \Omega$ (for $10 \mathrm{~A}$ ), and the dissipated power $10 \mathrm{~W}$. It is not easy to get high stability and low errors under these conditions. Besides, no galvanic isolation exists between input and output as it is obtained using current transformers. A 1000: 1 transformer ratio leads to a shunt resistor of $100 \Omega, 0.01 \mathrm{~W}$, which is possible to design with errors lower than 1 part in $10^{6}$ and phase displacements lower than 1 $\mu \mathrm{rad}$.

However, the difficulties of the $0.1 \Omega$ shunt design are then transferred to the design of the current transformer. There are some proposed methods to reduce the influence of the magnetizing current, which is the main cause of errors in current transformers at low frequencies. Some of them are based on double cores and double windings [1] and others on electronic compensating devices [2], [3]. But, if errors of one part in $10^{6}$ must be achieved in a transformer with 1000 turns, the stray capacitance effects cannot be ignored. It is proposed in this paper to use a shielded cable for the secondary winding. Generally, shields are connected to some guard potential, but the proposal here is to leave the shield potential floating.

\section{WINDING SHIELD}

It is common to use an electronic compensating device to reduce the magnetic flux in the core in order to minimize the magnetizing current. However, a significant voltage drop exists through the secondary winding caused by the secondary current. Its value depends on the secondary resistance $R$ and the secondary inductance (most cases negligible). So, the voltage

Manuscript received June 17, 2002; revised October 22, 2004.

The authors are with the UTE Laboratory (National Power Utility), 11800 Montevideo, Uruguay.

Digital Object Identifier 10.1109/TIM.2005.843565

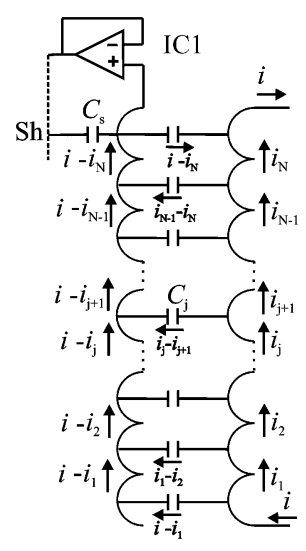

Fig. 1. Schematic diagram of the stray capacitances.

drop value $V_{c}$, in the secondary winding, can be enough to drive significant capacitive currents.

Since it is proposed to use a coaxial cable for the secondary winding, the inner conductor is free of stray capacitances between main turns. Instead, stray capacitances appear between the inner conductor and the shield and between different turns of the shield. However, the external conductor of the coaxial cable is nearly an equipotential electrode (because only small capacitive currents flows through it); then, the capacitive currents between different turns of the shield can be neglected. Fig. 1 shows a diagram of the winding. $C_{j}$ represents the cable capacitances (per turn) and $C_{s}$ the capacitance between the winding and a foil shield that covers the all-secondary winding. In this way, there are two currents flowing by each turn. One of them flows through the inner conductor $\left(i_{j}\right)$ and the other through the cable shield $\left(i-i_{j}\right)$. The total turn current is the sum of them, which is $i$, a constant value for all turns. This happens because the transformer output current $i$ cannot go outside the cable shield. This shows that each effective turn current (core current plus cable shield current) has the same value for all turns, equal to the output current. As both currents circulate by the same geometrical path, the situation is equivalent to a simple winding carrying a constant current where the effect of the capacitances $C_{j}$ was eliminated.

The shield potential will be the potential of the central point of the winding, because of the symmetry of the equivalent capacitive circuit. To avoid capacitive currents from the cable shield to any external object, a guard is necessary. An external foil-shield (Sh) is used, driven by the amplifier IC1. It acts as an active guard, assuring that no current flows between the cable shield and any external electrode (including the capacitance $C_{s}$ ). The current through the cable shield $I_{m}(x)$ can be calculated based on the voltage between the inner conductor and the shield $V(x)$, the capacitance per meter of the cable, $c$, and the total inner conductor voltage drop $V_{c} . x$ is the distance from one end of the 


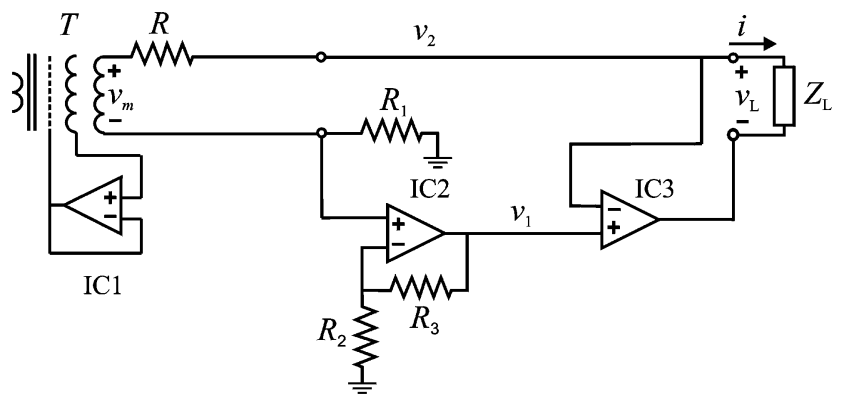

Fig. 2. Circuit of the proposed shielded compensated transformer.

winding to the considered point (total length: $l$ ). For this analysis, sinusoidal conditions were assumed, but similar results can be obtained for any current waveform. Then,

$$
V(x)=\frac{V_{c}}{2}\left(1-\frac{2 x}{l}\right)
$$

and

$$
I_{m}(x)=\int_{0}^{x} V(x) \omega c d x=\frac{V_{c}}{2} \omega c\left(x-\frac{x^{2}}{l}\right) .
$$

This parabolic shape has a maximum value at the center $(x=$ $l / 2$ ) and null values at both ends. Then, the voltage drop in the shield $V_{s}$ is

$$
V_{s}=\int_{0}^{L} r_{s} I_{m} d x=\frac{\omega R_{s} c l}{12} V_{c}
$$

where $r_{s}$ is the shield resistance per meter and $R_{s}$ its total value. For the example (see Section IV), $V_{s}=100 \mu \mathrm{V}$, at $1 \mathrm{kHz}$, around $10^{4}$ times smaller than the inner conductor voltage drop, which leads to interturn currents in the order of $10^{-9} \mathrm{~A}$. This justifies the assumption of an equipotential shield.

\section{General Design}

Fig. 2 shows the complete circuit. $T$, including $R$ (secondary winding resistance), is the current transformer. The primary consists of ten turns that can be connected in series or parallel (ratios $1: 100$ to $1: 1000$ ). The secondary has 1000 turns made of shielded cable. Its secondary inductance was not taken into account because its reactance was negligible. According to [3], the output current is sensed by the shunt resistor $R_{1}$, and this information is sent to the OpAmp IC2. On the other hand

$$
v_{m}=R i+v_{2}+R_{1} i \text {. }
$$

The OpAmp IC3 determines that voltage $v_{1}$ equals voltage $v_{2}$, for any load $Z_{L}$. Then

$$
v_{m}=\left(R-\frac{R_{1} R_{3}}{R_{2}}\right) \cdot i .
$$

To obtain a zero magnetizing voltage, the value of the ratio $R_{2} / R_{3}$ must be equal to $R_{1} / R$. This cancels the magnetizing current.

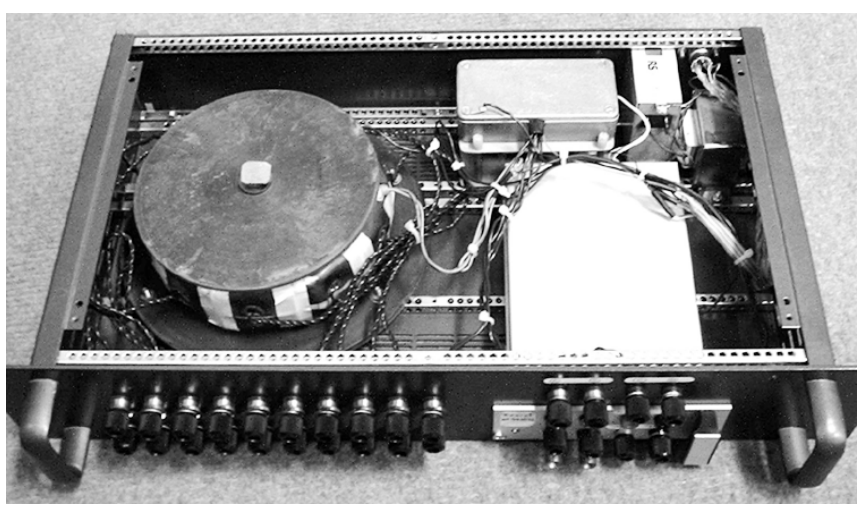

Fig. 3. Current transformer with shielded windings.

\section{APPLICATION EXAMPLE}

A current transformer with this design was built. It is used in the national power standard as the input current to voltage transducer. The parallel-series configurations of the primary lead to nominal input currents of 1, 2, 5, and $10 \mathrm{~A}$. Fig. 3 shows the general appearance of the device. The transformer itself is on the left, the electronic circuitry on the right at the back, and an $100 \Omega$ output resistor (load) at the front. The secondary winding was made with a very thin coaxial cable (diameter $1.5 \mathrm{~mm}$ ), with a resistance value of $43 \Omega$. Its voltage drop is around $0.43 \mathrm{~V}$ for $10 \mathrm{~mA}$ secondary current. Its total length is $150 \mathrm{~m}$, and the capacitance between the inner conductor and the shield is $200 \mathrm{pF} / \mathrm{m}$. These data lead to a maximum shield current of $10 \mu \mathrm{A}$ at $1 \mathrm{kHz}$ (at the central point of the winding shield), which produces a voltage drop of $100 \mu \mathrm{V}$ (resistance of the shield: $17 \Omega$ ).

The device was calibrated, comparing it against resistors. Two standard resistors of $1 \Omega$ and $100 \Omega$ were connected to the input and the output terminals of the transformer (100:1 ratio). The $100 \Omega$ resistor was made of four $100 \Omega$ individual Vishay resistors, and the $1 \Omega$, by 100 units of $100 \Omega$ noninductive resistors.

In this way, the voltage drop on each resistor has approximately the same value ( $1 \mathrm{~V}$ at the nominal current). To compare these voltage drops, the low potential points of both resistors were connected together, and the voltage difference was amplified and then measured by a Fourier analyzer acting as a zero detector. A bridge was completed, adding a variable capacitor and a variable resistor in parallel with the 1 and $100 \Omega$ standard resistors. When the bridge achieves the equilibrium, the value of the variable resistor gives the transformer ratio error and the value of the capacitor, the transformer phase shift. The estimated uncertainties of the measurement are $2 \mu \mathrm{A} / \mathrm{A}$ and $2 \mu \mathrm{rad}$.

The measured errors are shown in Table I. At $50 \mathrm{~Hz}$, they are $-1.8 \mu \mathrm{A} / \mathrm{A}$ and $0.3 \mu \mathrm{rad}$, and at $1 \mathrm{kHz}$ they are $-0.2 \mu \mathrm{A} / \mathrm{A}$ and $0.0 \mu \mathrm{rad}$. These values include all error sources. The main sources are the magnetizing current compensation (associated to the electronic device), the standard resistors and the shield technique. With this transformer-error-measurement technique, it is not possible to measure the errors that come only from the proposed shield technique. To achieve this goal, a new autocalibrating shielded transformer is being designed. 
TABLE I

TRANSFORMER ERRORS AT OUTPUT CURRENT OF 50 MA

\begin{tabular}{|c|c|c|}
\hline \multirow{2}{*}{$\begin{array}{c}\text { Frequency } \\
(\mathrm{Hz})\end{array}$} & \multicolumn{2}{|c|}{ Errors } \\
\hline & $\begin{array}{c}\text { Ratio } \\
(\mu \mathrm{A} / \mathrm{A})\end{array}$ & $\begin{array}{c}\text { Phase shift } \\
(\mu \mathrm{rad})\end{array}$ \\
\hline 50 & -1.8 & 0.3 \\
\hline 100 & 2.9 & 0.6 \\
\hline 200 & 2.9 & 1.2 \\
\hline 400 & 0.4 & 2.4 \\
\hline 700 & -0.2 & 2.2 \\
\hline 1000 & -0.2 & 0.0 \\
\hline
\end{tabular}

\section{CONCLUSION}

A compensated current transformer was described. An electronic device reduces the magnetizing current, so that the following relevant error source is the parasitic interturn capacitances. To null this effect, a shielded winding is proposed. No guard potential is applied to the shield. Instead of that, the shield is floating and its potential is defined by the capacitances between it and the inner conductor of the cable. This work shows that, in this way, the errors coming from that source are greatly reduced. An experimental test shows errors lower than $3 \mu \mathrm{A} / \mathrm{A}$ and $3 \mu \mathrm{rad}$ between $50 \mathrm{~Hz}$ and $1 \mathrm{kHz}$. To evaluate more precisely the potential of the proposed shielding method, the measurement uncertainties must be reduced. It is planned to develop a self calibrating current transformer, using the proposed technique, to avoid the need of standard resistors for the error measurement, and to avoid errors coming from the magnetizing current compensation (which are the most important sources of uncertainty).

\section{REFERENCES}

[1] N. L. Kusters and W. J. M. Moore, "The compensated current comparator; a new reference standard for current-transformer calibrations in industry," IEEE Trans. Instrum. Meas., vol. IM-13, pp. 107-114, Jun.-Sep. 1964.

[2] T. M. Souders, "Wide-band two-stage current transformers of high accuracy," IEEE Trans. Instrum. Meas., vol. IM-21, pp. 340-349, Nov. 1972.

[3] D. Slomovitz, "Electronic error reduction system for clamp-on probes and measuring current transformers," IEEE Trans. Instrum. Meas., vol. 49, no. 6, pp. 1278-1281, Dec. 2000. 